\title{
The natural course of passive tenodesis grip in individuals with spinal cord injury with preserved wrist extension power but paralyzed fingers and thumbs
}

\author{
Hae Yoon Jung ${ }^{1,2} \cdot$ Jieun Lee ${ }^{3} \cdot$ Hyung Ik Shin ${ }^{2}$ \\ Received: 21 November 2017 / Revised: 23 March 2018 / Accepted: 29 March 2018 / Published online: 22 May 2018 \\ (c) International Spinal Cord Society 2018
}

\begin{abstract}
Study design Cross-sectional.

Objectives To investigate the natural course of passive tenodesis grip in individuals with spinal cord injury (SCI) with no experience of tenodesis splint application and the related factors for success of the grip.

Setting Community-dwelling persons with chronic SCI in South Korea.

Methods Individuals with cervical SCI with preserved wrist extensor power, but completely paralyzed fingers and thumbs, were recruited. For each hand, success or failure of passive tenodesis lateral grip was assessed both in the opening and closing phase. The key task in the Graded and Redefined Assessment of Strength, Sensibility, and Prehension (GRASSP) test was also assessed.

Results Fifty-eight hands of 37 individuals with SCI were analyzed. In 35 of the 58 hands, both opening and closing phases of the grip were achieved. During the closing phase of the passive tenodesis lateral grip, the mean (s.d.) value of the second MP joint flexion angle $\left(^{\circ}\right)$ in the success group was 55.1 (13.6), compared to $38.6(17.8)$ in the failure group. The key task in GRASSP was completed in only 14 out of 58 hands. Hands with a wrist extensor power of grade 4 or 5 on a manual muscle test showed higher GRASSP scores than those with a grade 3 wrist extensor power.

Conclusions The passive tenodesis lateral grip can be achieved in a large number of hands without splint application after SCI, but its use in the key task of the GRASSP is limited.
\end{abstract}

\section{Introduction}

One of the most devastating aspects of a cervical spinal cord injury (SCI) is the impairment of arm and hand function, which has a great impact on an individual's level of independence. Improving arm and hand function has been reported to be a high priority for individuals with SCI [1-4].

Non-orthotic passive tenodesis grip is used to alleviate hand function impairment in people with tetraplegia with

Hyung Ik Shin

hyungik1@snu.ac.kr

1 Department of Rehabilitation Medicine, Incheon Workers Compensation Hospital, Seoul, South Korea

2 Department of Rehabilitation Medicine, Seoul National University Hospital, Seoul, South Korea

3 Division of Occupational Therapy, Yeeun Rehabilitation Hospital, Bucheon, South Korea innervated wrist extensor muscles but paralyzed finger and thumb flexor muscles [5, 6]. This tenodesis grip occurs because the tendons of extrinsic finger and thumb flexor muscles pass over the wrist. When the wrist is actively extended, passive tension of the flexor muscles puts them under tension, creating a grip pattern between the thumb and fingers. Conversely, extending the fingers and thumb can be done by flexing the wrist to relieve passive tension of the flexor muscles. Typically, with tenodesis grip, the thumb points toward the radial side of the index finger, resulting in a lateral pinch $[5,6]$.

Because the effectiveness of the tenodesis grip is influenced by the passive properties of the hands, several splinting strategies to augment this mechanism have been applied, especially during the acute and post-acute phases after SCI; (1) Adaptive shortening of the flexor digitorum profundus, flexor digitorum superficialis, and the flexor pollicis longus muscles while maintaining finger joint range of motion, (2) stiffening of the thumb interphalangeal (IP) joint into neutral position, and (3) preventing laxity of the 
Fig. 1 Assessment of tenodesis lateral grip in the opening (a) and closing (b) phase
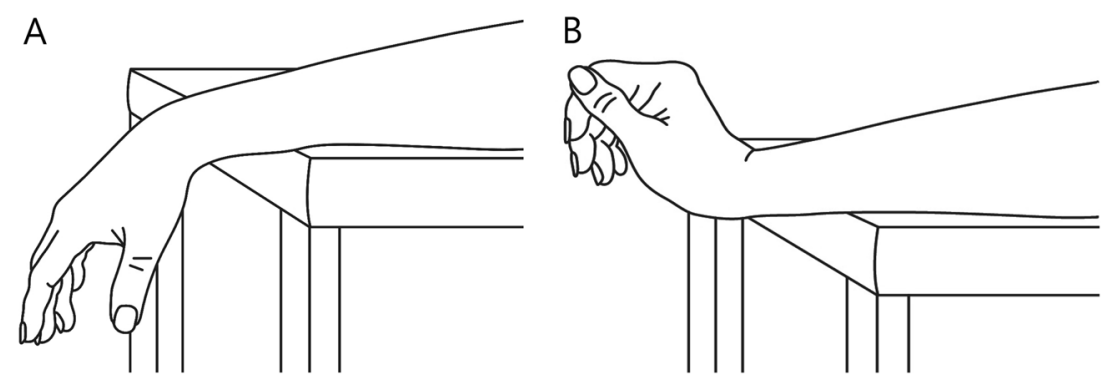

1st metacarpophalangeal (MP) joint and carpometacarpal (CMC) joint [7-9].

For these purposes, various static and dynamic tenodesis splints have been applied by different SCI centers [5-9]. For example, Harvey [5] suggested an alternative splinting protocol; one for the opening phase and the other for the closing phase of the grip. For the opening phase splint, the wrist was held in $45^{\circ}$ flexion, the second finger MCP joint in $90^{\circ}$ flexion, and the thumb and second finger IP joints held in full extension. For the closing phase splint, the wrist was held in $45^{\circ}$ extension, the second finger MCP and IP joints were held in $90^{\circ}$ flexion, and the thumb IP joint held in full extension.

Although several authors [5-7] suggested that these splints could enhance the effectiveness of passive tenodesis grip, there have been few reports providing evidence regarding the benefits of splinting or suggesting technical considerations for fabrication and application.

The aims of this study were to describe the natural course of the passive tenodesis grip in individuals with SCI who had no experience of splint application and to investigate related factors for a successful grip. The results of our study could serve as comparative data for future studies investigating the effectiveness and technical aspects of splint fabrication and application.

\section{Methods}

\section{Participants}

Participants of this study were recruited using a leaflet distributed in community wellness centers and physical training facilities frequently used by individuals with disabilities.

The following inclusion criteria were applied.

- Cervical SCI with paralysis in the hands

- Time from onset of more than 1 year

- No experience of upper extremity splint application for any purpose reported by the participants

- No combined peripheral or central nervous system disease or injury involving the upper extremity
In total, 174 community-dwelling individuals with cervical SCI were recruited and screened for this study. The participants agreed to the purpose and procedures of this study and completed a consent form. The hands of the participants were included for analysis if they were compatible with the following criteria.

- Grade 3 or higher on the wrist extensor manual muscle test

- No palpable or observable contraction in both intrinsic and extrinsic hand muscles

A neurologic examination was performed using the International Standards for Neurological Classification of SCI for participants who had at least one hand analyzed for this study. The institutional review board of Seoul National University Hospital approved this study (1504-023-662), and all the methods adhered to the principles of the Declaration of Helsinki.

\section{Assessment of passive tenodesis grip}

The participants placed their forearms on the table with their palms facing the floor. In this position, the participants were requested to relax their wrists, which resulted in gravityassisted wrist palmar flexion. Subsequently, they were asked to maximally extend their wrists. As shown in Fig. 1, voluntary wrist extension causes CMC joint flexion, first MP joint flexion, thumb IP joint flexion, second MP joint flexion, second proximal interphalangeal (PIP) joint flexion, and second distal interphalangeal (DIP) joint flexion simultaneously, which directs the thumb to the index finger, resulting in lateral grip.

When there was space between the thumb and index finger yielded by gravity-assisted wrist palmar flexion, the opening phase of the passive tenodesis grip was regarded as successful. However, when there was no space between the thumb and index finger through wrist palmar flexion, the opening phase of the grip was regarded as a failure. When the thumb touched any part of the second finger with maximal voluntary wrist dorsiflexion, the closing phase of the grip was regarded as successful. When the thumb did not touch the second finger, the closing phase of the grip was regarded as a failure. 


\section{Assessment of thumb and finger joint angle}

In both the opening and closing phases of passive tenodesis grip, the thumb IP, thumb MP, first CMC, index DIP, index finger PIP, and index finger MP joint angles were measured using a finger goniometer. An occupational therapist with 10 years of experience working with individuals with SCI performed the measurements. Each joint was measured twice and the results were averaged.

\section{Assessment of the key task}

To measure the hand capacity of the participants, a key task was assessed using the Graded and Redefined Assessment of Strength, Sensibility and Prehension (GRASSP) version 1.0. The GRASSP is a quantitative and qualitative clinical upper limb impairment measure designed for use in individuals with acute and chronic cervical SCI and the key task is one of the six subtests in the GRASSP $[10,11]$. The test board was placed on the edge of the table and the key was also placed on the same table in front of the participant. The task was completed when the participant picked up the key, inserted it into a lock, and rotated it 90 degrees.

The following scoring system was used.

0 - The task cannot be performed at all

1 - The task cannot be completed (less than $50 \%$ of the task completed)

2 - The task cannot be completed (50\% or more of the task completed)

3 - The task is completed using tenodesis or an alternative grasp other than the expected grasp.

4 - The task is conducted using the expected grasp with difficulty (lack of smooth movement or difficult and slow movement)

5 - The task is conducted without difficulties using the expected grasping pattern

Fifty percent of the task was defined when the participant was able to get the key into the insertion point in the lock. One minute was allowed for the task.

\section{Statistical analysis}

Differences between the lateral grip success and failure groups regarding finger joint angle were compared with an independent $t$-test. Group differences in GRASSP scores were compared via the Mann-Whitney $U$ test because the GRASSP score could be regarded as an ordinal variable. All data analyses were conducted using SPSS 19 software (SPSS Inc., Chicago, IL, USA). The level of significance was set at $P<0.05$.
Table 1 Participant characteristics

\begin{tabular}{|c|c|c|c|}
\hline Variables & Group & $N$ & Rate $(\%$ \\
\hline \multirow[t]{2}{*}{ Sex } & Male & 35 & 95 \\
\hline & Female & 2 & 5 \\
\hline \multirow[t]{7}{*}{ Etiology of injury } & Traumatic & & \\
\hline & Transport & 14 & 38 \\
\hline & Fall & 17 & 46 \\
\hline & $\begin{array}{l}\text { Diving in } \\
\text { shallow water }\end{array}$ & 3 & 8 \\
\hline & Other injury & 2 & 5 \\
\hline & Nontraumatic & & \\
\hline & Iatrogenic & 1 & 3 \\
\hline \multirow[t]{12}{*}{ Neurological classification $^{\mathrm{a}}$} & C4 & & \\
\hline & AIS C & 1 & 3 \\
\hline & $C 5$ & & \\
\hline & AIS A & 4 & 11 \\
\hline & $\mathrm{B}$ & 9 & 24 \\
\hline & $\mathrm{C}$ & 3 & 8 \\
\hline & C6 & & \\
\hline & AIS A & 11 & 30 \\
\hline & $\mathrm{B}$ & 6 & 16 \\
\hline & $\mathrm{C}$ & 2 & 5 \\
\hline & $C 7$ & & \\
\hline & AIS A & 1 & 3 \\
\hline
\end{tabular}

AIS American Spinal Injury Association Impairment Scale

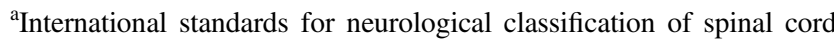
injury

\section{Results}

\section{Participant characteristics}

In total, 290 hands out of 348 hands were excluded from analysis because residual voluntary finger and thumb movements were palpable or observable. Fifty-eight hands (30 right hands and 28 left hands) of 37 individuals with SCI were included for analysis. The characteristics of the participants are described in Table 1. The mean (s.d.) age was 47.8 (16.1) years, and the median (IQR) time from onset was 38 (25-46) months.

\section{Passive tenodesis grip}

In 35 of the 58 hands (60\%), both phases of opening and closing were achieved. Sixteen of the 58 hands failed to create space between the thumb and index finger. No space was created in eleven hands, while the thumb was under the index finger obliterating the first web space in five hands (Fig. 2a). Thirty-seven of the 58 hands (64\%) succeeded in the closing phase of passive tenodesis grip. The thumb touched the second finger's distal phalanx/DIP joint in 
Table 2 Finger joint angle $\left(^{\circ}\right)$ at opening and closing phase of tenodesis lateral grip

\begin{tabular}{llllllll}
\hline Performance & Attainment & $\begin{array}{l}\text { CMC } \\
\text { flexion }\end{array}$ & $\begin{array}{l}\text { 1st MP } \\
\text { flexion }\end{array}$ & $\begin{array}{l}\text { Thumb IP } \\
\text { flexion }\end{array}$ & $\begin{array}{l}\text { 2nd MP } \\
\text { flexion }\end{array}$ & $\begin{array}{l}\text { 2nd PIP } \\
\text { flexion }\end{array}$ & $\begin{array}{l}\text { 2nd DIP } \\
\text { flexion }\end{array}$ \\
\hline Opening & $\begin{array}{l}\text { Success } \\
(N=42)\end{array}$ & $38.7 \pm 9.5$ & $17.6 \pm 10.3$ & $20.0 \pm 15.7$ & $50.3 \pm 18.3$ & $36.9 \pm 19.5$ & $17.3 \pm 15.2$ \\
& $\begin{array}{l}\text { Fail } \\
(N=16)\end{array}$ & $36.3 \pm 6.2$ & $22.2 \pm 13.5$ & $19.9 \pm 15.3$ & $45.4 \pm 18.0$ & $40.6 \pm 15.9$ & $17.2 \pm 11.7$ \\
& & & & & & \\
Closing & $\begin{array}{l}\text { Success } \\
(N=37)\end{array}$ & $38.5 \pm 8.1$ & $18.8 \pm 11.2$ & $18.6 \pm 13.3$ & $55.1 \pm$ & $37.5 \pm 17.9$ & $17.1 \pm 13.4$ \\
& & & & $13.6^{\mathrm{a}}$ & & \\
& Fail & $36.9 \pm 8.6$ & $19.6 \pm 10.9$ & $24.9 \pm 17.3$ & $38.6 \pm$ & $38.2 \pm 16.0$ & $16.4 \pm 13.8$ \\
& $(N=21)$ & & & & $17.8^{\mathrm{a}}$ & & \\
\hline
\end{tabular}

Data are presented as mean \pm s.d.

$C M C$ carpometacarpal joint, $M P$ metacarpal joint, $I P$ interphalangeal joint, $P I P$ proximal interphalangeal joint, $D I P$ distal interphalangeal joint

${ }^{\mathrm{a}} p<0.01$
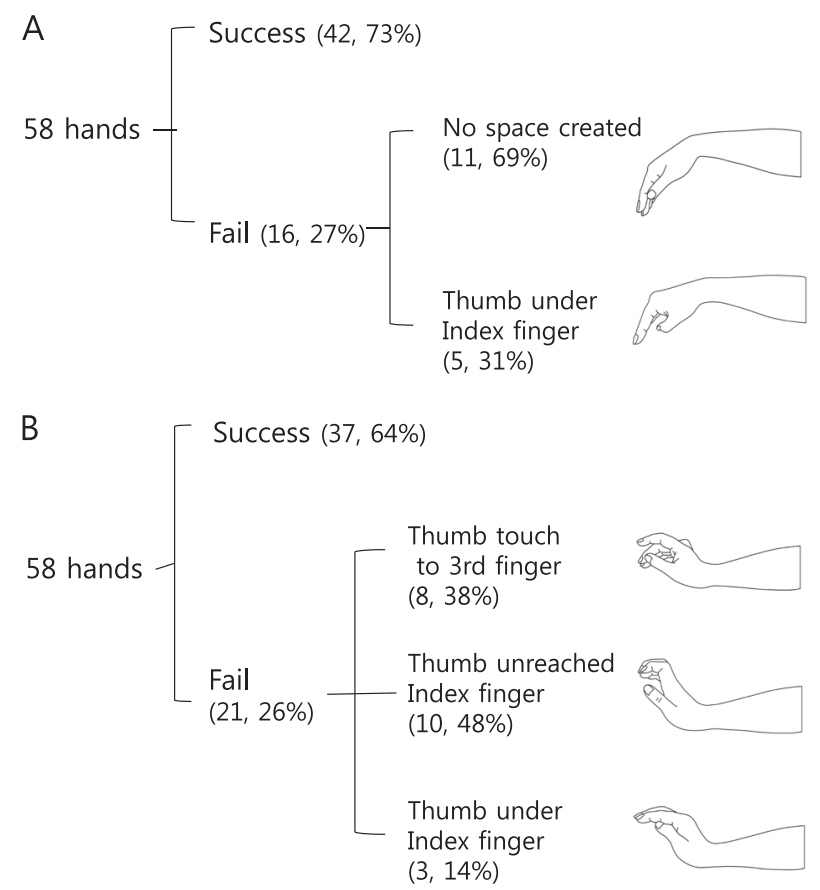

Fig. 2 Profiles of tenodesis lateral grip in the opening (a) and closing (b) phase

10 hands, middle phalanx/PIP joint in 22 hands, and proximal phalanx in 5 hands. The thumb touched the third finger in 8 hands (Fig. 2b). The thumb did not reach the index finger in 10 hands, while in 3 hands, the thumb was located under the index finger (Fig. 2b).

\section{Thumb and finger joint angles}

The CMC, first MP, thumb IP, second MP, second PIP, and second DIP joint angles at wrist flexion and extension are presented in Table 2. During the closing phase of the passive tenodesis lateral grip, the mean (s.d.) value of the second MP joint flexion angle $\left(^{\circ}\right)$ in the success group was 55.1 (13.6), compared to 38.6 (17.8) in the failure group ( $t=4.654, p=0.000)$. However, in the opening phase, there were no differences in finger joint angles between the success and failure groups.

\section{GRASSP score}

The key task in GRASSP was completed in 14 out of 58 hands (24\%) when splints were not applied to augment passive tenodesis grip (Fig. 3).

The closing phase success group showed a higher GRASSP score than the closing phase failure group $(Z=-2.924, p=0.003)$. However, there were no differences between the opening failure and success groups in GRASSP score. Hands with a wrist extensor power of grade 4 or 5 in the manual muscle test showed higher GRASSP scores than those with grade $3(Z=-3.656, p=0.000)$.

\section{Discussion}

After SCI resulting in paralyzed fingers and thumbs but preserved wrist extension power, temporary use of a splint has been advised to achieve effective passive tenodesis lateral grip [5, 6]. In this study, more than half of the hands attained the grip without splint application. Thirty-five of 58 hands $(60 \%)$ achieved both opening and closing phases of the grip and the closing phase of the grip was achieved in 37 of 58 hands (64\%). These results could be explained by stiffness and/or spasticity of paralyzed extrinsic thumb and finger flexor muscles, which spontaneously developed in a more functional direction without splint application after SCI. On the contrary, in only 14 out of 58 hands (24\%), the key task in GRASSP was completed (Fig. 3).

This result could be explained at least partially by the pinch force produced by the passive tenodesis grip, which was reported to be extremely small and indistinguishable from no force when a standard clinical pinch meter was used $[12,13]$. Most activities of daily living (e.g., stabbing 


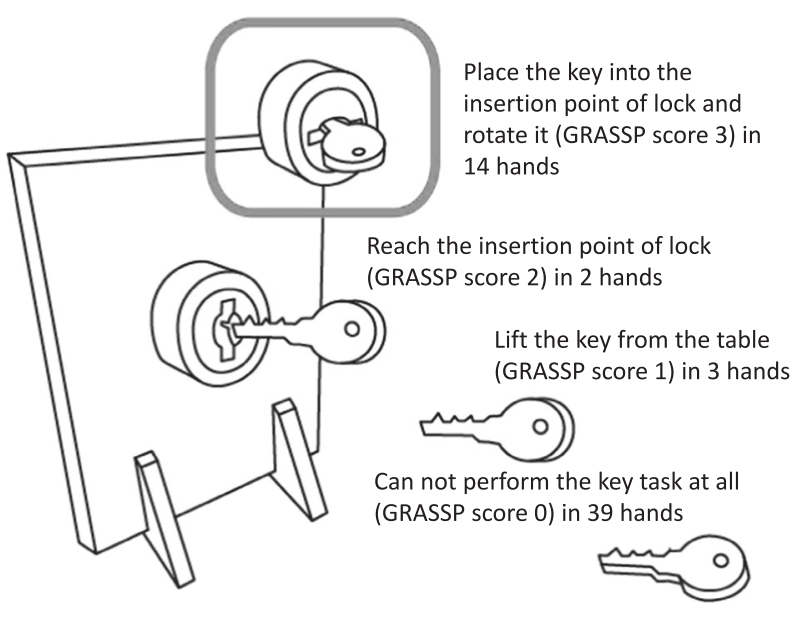

Fig. 3 The results of the key task in Graded and Redefined Assessment of Strength, Sensibility and Prehension (GRASSP) test

food with fork) performed by individuals with tetraplegia require a pinch force $>2$ Newton [14]. In addition, voluntary movements of ulnar or radial deviation at the wrist could be needed to get the key into the lock, since the GRASSP test board was placed parallel to the table edge and was not allowed to be at an angle. This might explain why the three participants who were able to lift the key from the table failed to reach the insertion point of the lock (Fig. 3).

There was another limitation in the passive tenodesis grip: no participant in this study acquired tip-to-tip or cylindrical grip. Typically, the lateral key grip is the only grip pattern that can be obtained using passive tenodesis effects, although several authors suggest that the tip-to-tip pinch rarely can be obtained with a long adductor pollicis muscle $[5,6]$. On the contrary, through grip reconstructive surgery, cylindrical and tip-to-tip pinch functions also can be acquired or reinforced [15] and pinch force can be strengthened up to $2 \mathrm{~kg}$ [16].

Therefore, the results of this study suggest the necessities for discussions with individuals with SCI regarding the goals and limitations of splint application for passive tenodesis grip and tendon transfer surgery, which can supplement the limitations of the grip [17].

There were no differences between the opening phase success and failure groups in the GRASSP score. Participants commonly compensated the opening phase of tenodesis grip by placing their thumbs on the exam table and dragging them to the palmar side of the hand, creating a space between the thumb and index finger. Although this alternative technique to create the space between thumb and index finger was less applicable to other tasks in GRASSP, such as the cylindrical grasp and spherical grasp, this technique was allowed because the GRASSP 1.0 manual does not give specific guidance regarding the issue.
We also observed several alternatives in the closing phase of the grip accommodated by motivated patients. For example, the authors observed the interwoven pattern that a key is put on the third finger and fastened by the second and fourth fingers, and the interdigital pattern that a key is put between the second and third fingers. However, we did not allow these compensated methods during the GRASSP test because this study focused on the passive tenodesis lateral grip. In the GRASSP version 1.0, any grasp pattern is allowed, and when the key task is completed using tenodesis or an alternative grasp other than the expected grasping pattern, it is regarded as a score of 3 . In eight hands in this study, the thumb moved beneath the second finger and touched the third finger in the closing phase as shown in Fig. 2b. In those variation cases, we observed that the MP and IP joints of the third finger were in a more flexed position compared to the second finger in the closing phase, and as a result, could play the role as a back-up finger when usual passive tenodesis lateral grip to the second finger failed. Some individuals might be satisfied with this variation from a lateral grip even though the key task in the GRASSP test failed. The individuals' perspectives on their grip pattern should be included in future studies that investigate the effects of a splint program on hand posture and function in individuals with tetraplegia.

Finger joint angles were different between the success and failure groups only in the second finger MP joint in the closing phase of passive tenodesis grip. This result suggests that second MP joint flexion with the wrist extended is the most essential component of the splinting procedure for passive tenodesis grip after SCI.

Hands with a wrist extensor power of grade 4 or 5 in the manual muscle test recorded a higher GRASSP score than those with grade 3 . With stronger wrist extensors, a firmer passive tenodesis grip could be accomplished. Additionally, the strength of passive tenodesis lateral grip could be adjusted by exerting a different level of wrist extensor force in the hand with a wrist extensor power of grade 4 or 5 resulting in more functional use of hand. This finding suggests that strengthening of the wrist extensor muscles could have functional implications for enhancing the use of passive tenodesis grip in individuals with SCI with preserved wrist extensors but paralyzed intrinsic and extrinsic hand muscles.

Several limitations of this study should be acknowledged. First, we only evaluated the attainment of lateral grip by passive tenodesis effect, and therefore, we did not know how this grip function would be translated into functional use of the hands [18, 19]. There are further variables that could influence functional use of the hand, including sensory function of the hands [20], muscle strength [21-23], types of grasp other than tenodesis lateral prehension [23, 24], and functional training [25]. In the future, longitudinal studies should investigate the effectiveness of splinting 
regimens on the functional use of hands in individuals with SCI. Second, only individuals with tetraplegia with complete paralysis in their fingers and thumb were analyzed in this study. However, in actual clinical situations, the majority of individuals with SCI would have partially preserved intrinsic or extrinsic hand muscle power. The suggestions obtained from this study should be tailored and individualized according to the residual motor function and individual characteristics of the person. Third, participants in this study were recruited using a leaflet distributed in community-based facilities rather than, for example, enrolling a consecutive series of patients admitted to a hospital. Therefore, there might be a selection bias of recruiting more motivated and active individuals with SCI, which could affect the results of this study. Fourth, in this study, one minute was allowed for the completion of the key task, which was shorter than $75 \mathrm{~s}$ in GRASSP version 1.0 instruction. This deviation from the standardized administration of the test could affect the results of this study, underestimating the capacity of the participants.

\section{Conclusions}

The passive tenodesis lateral grip can be achieved in a large number of hands without splint application after SCI, but its use in the key task of the GRASSP is limited. Maintaining the second MP joint angle in a flexed posture when the wrist is extended using a splint and strengthening the wrist extensor muscles could have functional implications to enhance the effectiveness of passive tenodesis grip in individuals with SCI with preserved wrist extensor but paralyzed fingers and thumbs.

Authors contribution HYJ was responsible for acquisition of data, analysis, and interpretation of data, and writing the protocol. LJ was responsible for acquisition of data, conducting the search, and making the study concept and design. HIS was responsible for making the study concept and design and drafting the work, and critical revision of the manuscript.

Funding This work was funded by Korea Workers' Compensation and Welfare Service. This study was funded by Seoul National University Hospital Grant No. 0420150650 (2015-1218).

\section{Compliance with ethical standards}

Conflict of interest The authors declare that they have no conflict of interest.

\section{References}

1. Lu X, Battistuzzo CR, Zoghi M, Galea MP. Effects of training on upper limb function after cervical spinal cord injury: a systematic review. Clin Rehabil. 2015;29:3-13.
2. Snoek GJ, IJzerman MJ, Hermens HJ, Maxwell D, BieringSorensen F. Survey of the needs of patients with spinal cord injury: impact and priority for improvement in hand function in tetraplegics. Spinal Cord. 2004;42:526-32.

3. Anderson KD. Targeting recovery: priorities of the spinal cordinjured population. J Neurotrauma. 2004;21:1371-83.

4. Simpson LA, Eng JJ, Hsieh JT, Wolfe DL. The health and life priorities of individuals with spinal cord injury: a systematic review. J Neurotrauma. 2012;29:1548-55.

5. Harvey L. Principles of conservative management for a nonorthotic tenodesis grip in tetraplegics. $\mathbf{J}$ Hand Ther. 1996;9:238-42.

6. Johanson ME, Murray WM. The unoperated hand: the role of passive forces in hand function after tetraplegia. Hand Clin. 2002;18:391-8.

7. Curtin M. Development of a tetraplegic hand assessment and splinting protocol. Paraplegia. 1994;32:159-69.

8. DiPasquale-Lehnerz P. Orthotic intervention for development of hand function with C-6 quadriplegia. Am J Occup Ther. 1994;48:138-44.

9. Krajnik SR, Bridle MJ. Hand splinting in quadriplegia: current practice. Am J Occup Ther. 1992;46:149-56.

10. Kalsi-Ryan S, Beaton D, Ahn H, Askes H, Drew B, Curt A, et al. Responsiveness, sensitivity, and minimally detectable difference of the graded and redefined assessment of strength, sensibility, and prehension, version 1.0. J Neurotrauma. 2016;33:307-14.

11. Kalsi-Ryan S, Curt A, Verrier MC, Fehlings MG. Development of the Graded Redefined Assessment of Strength, Sensibility and Prehension (GRASSP): reviewing measurement specific to the upper limb in tetraplegia. J Neurosurg Spine. 2012;17(Suppl 1):65-76.

12. McCarthy CK, House JH, Van Heest A, Kawiecki JA, Dahl A, Hanson D. Intrinsic balancing in reconstruction of the tetraplegic hand. J Hand Surg Am. 1997;22:596-604.

13. Van Heest A, Hanson D, Lee J, Wentdorf F, House J. Split flexor pollicus longus tendon transfer for stabilization of the thumb interphalangeal joint: a cadaveric and clinical study. J Hand Surg Am. 1999;24:1303-10.

14. Smaby N, Johanson ME, Baker B, Kenney DE, Murray WM, Hentz VR. Identification of key pinch forces required to complete functional tasks. J Rehabil Res Dev. 2004;41:215-24.

15. Dunn JA, Sinnott KA, Rothwell AG, Mohammed KD. Simcock JW4. Tendon transfer surgery for people with tetraplegia: an overview. Arch Phys Med Rehabil. 2016;97:S75-80.

16. Hamou C, Shah NR, DiPonio L, Curtin CM. Pinch and elbow extension restoration in people with tetraplegia: a systematic review of the literature. J Hand Surg Am. 2009;34:692-9.

17. Dunn JA, Hay-Smith EJ, Whitehead LC, Keeling S. Issues influencing the decision to have upper limb surgery for people with tetraplegia. Spinal Cord. 2012;50:844-7.

18. Harvey LA, Batty J, Jones R, Crosbie J. Hand function of C6 and C7 tetraplegics 1 - 16 years following injury. Spinal Cord. 2001;39:37-43.

19. Kalsi-Ryan S, Beaton D, Curt A, Duff S, Jiang D, Popovic MR, et al. Defining the role of sensation, strength, and prehension for upper limb function in cervical spinal cord injury. Neurorehabil Neural Repair. 2014;28:66-74.

20. Curt A, Dietz V. Traumatic cervical spinal cord injury: relation between somatosensory evoked potentials, neurological deficit, and hand function. Arch Phys Med Rehabil. 1996;77:48-53.

21. Ota T, Akaboshi K, Nagata M, Sonoda S, Domen K, Seki M, et al. Functional assessment of patients with spinal cord injury: measured by the motor score and the functional independence measure. Spinal Cord. 1996;34:531-5. 
22. Rudhe C, van Hedel HJ. Upper extremity function in persons with tetraplegia: relationships between strength, capacity, and the spinal cord independence measure. Neurorehabil Neural Repair. 2009;23:413-21.

23. Velstra IM, Bolliger M, Krebs J, Rietman JS, Curt A. Predictive value of upper limb muscles and grasp patterns on functional outcome in cervical spinal cord injury. Neurorehabil Neural Repair. 2016;30:295-306.
24. Mateo S, Revol P, Fourtassi M, Rossetti Y, Collet C, Rode G. Kinematic characteristics of tenodesis grasp in C6 quadriplegia. Spinal Cord. 2013;51:144-9.

25. Spooren AI, Janssen-Potten YJ, Kerckhofs E, Seelen HA. Outcome of motor training programmes on arm and hand functioning in patients with cervical spinal cord injury according to different levels of the ICF: a systematic review. J Rehabil Med. 2009;41:497-505. 